\title{
Generating human intestinal tissue from pluripotent stem cells in vitro
}

\author{
Kyle W. McCracken ${ }^{1}$, Jonathan C. Howell ${ }^{2}$, James M. Wells ${ }^{*}, 1$, and Jason R. Spence ${ }^{1,3}$ \\ Kyle W. McCracken: Kyle.mccracken@cchmc.org; Jonathan C. Howell: Jonathan.howell@cchmc.org; Jason R. Spence: \\ Jason.spence@cchmc.org \\ ${ }^{1}$ Division of Developmental Biology, Cincinnati Children's Hospital Medical Center, 3333 Burnet \\ Avenue, Cincinnati, Ohio 45229-3039, USA \\ 2Division of Endocrinology, Cincinnati Children's Hospital Medical Center, 3333 Burnet Avenue, \\ Cincinnati, Ohio 45229-3039, USA \\ ${ }^{3}$ Division of Gastroenterology, Hepatology and Nutrition, 3333 Burnet Avenue, Cincinnati, Ohio \\ 45229-3039, USA
}

\section{Abstract}

We describe a protocol to generate 3-dimensional human intestinal tissue (called organoids) in vitro from human pluripotent stem cells. To generate intestinal organoids, pluripotent stem cells are first differentiated into FOXA2+/SOX17+ endoderm by treating the cells with ActivinA for 3 days. Following endoderm induction, the pluripotent stem cells are patterned into CDX2+ mid/ hindgut tissue using FGF4 and WNT3a. During this patterning step, 3-dimensional mid/hindgut spheroids bud from the monolayer epithelium attached to the tissue culture dish. The 3dimensional spheroids are further cultured in matrigel along with pro-intestinal growth factors, and proliferate and expand over 1-3 months to give rise to intestinal tissue, complete with intestinal mesenchyme and epithelium consisting of all of the major intestinal cell types. To date, this is the only method to efficiently direct differentiation of human pluripotent stem cells into 3dimensional human intestinal tissue in vitro.

\section{Keywords}

Human; intestine; stem cells; pluripotent; differentiation; endoderm; CDX2; hindgut; development

\section{Introduction}

\section{Development of the protocol}

\begin{abstract}
A critical but often underappreciated step in the development of vertebrates is the early patterning that occurs during and after gastrulation. These patterning events set up the anterior-posterior (A-P) and dorsal-ventral axes of the embryo. A-P patterning of the endoderm germ layer establishes foregut (anterior) and mid/hindgut (posterior) domains. The mid and hindgut gives rise to the small and large intestines ${ }^{1}$. Studies using several
\end{abstract}

\footnotetext{
*Author for correspondence james.wells@cchmc.org, Fax: 513-636-4317, Phone: 513-636-8767. Author Contributions Statement

J.M.W., J.R.S., J.C.H., and K.W.M. conceived the study and experimental design. J.M.W. and J.R.S. analyzed data and co-wrote the manuscript. J.C.H. and K.W.M. performed experiments.

Competing Financial Interests Statement

J.M.W. and J.R.S. are inventors on a patent involving the system described herein.
} 
model organisms demonstrate that the FGF and WNT signaling pathways promote posterior patterning and specification of hindgut endoderm ${ }^{2-4}$. We hypothesized that the ability of FGF and/or WNT signaling to promote posterior endoderm patterning could be exploited to generate posterior mid- and hindgut lineages from human pluripotent stem cells (hPSCs). In support of this, we recently published a method that demonstrates that high levels of FGF4 and WNT3A act in synergy to pattern human PSC-derived definitive endoderm into mid/ hindgut endoderm and promote a gut tube-like morphogenesis, resulting in the formation of 3-dimensional, mid/hindgut-like spheroids ${ }^{5}$. We then utilized a culture system that promotes expansion of adult intestinal stem cells ${ }^{6}$ to expand mid/hindgut spheroids into 3-

demensional human intestinal tissue in culture.

We have demonstrated that formation of human intestinal tissue from PSCs is via a process that very closely mimics embryonic intestine development, giving rise to an intestinal epithelium containing absorptive enterocytes, as well as the major secretory lineages including Paneth cells, goblet cells and enteroendocrine cells. The human intestinal tissue is functional as it can secrete mucins into luminal structures, and can absorb fluorescently labeled dipeptides, suggesting a functional dipeptide transport system ${ }^{7,8}$. Intestinal tissues contain villus-like structures with microscopic brush borders as well as crypt-like structures that express intestinal stem cell markers. Lastly, we have demonstrated that this is a genetically tractable system that allows for both genetic gain- and loss-of function studies.

\section{Experimental Design}

Generation of human intestinal tissue from hPSCs takes approximately one month: 3 days of exposure to ActivinA for definitive endoderm (DE) induction, 4 days of exposure to FGF4/ WNT3A to generate mid/hindgut spheroids, and 14-28 days to allow spheroids to expand into intestinal tissue. During each of these steps, genetic manipulation and addition/removal of growth factors and/or chemical agonists and antagonists is possible to achieve different experimental outcomes. At each step, the appropriate controls should be included: during DE induction, a control excluding ActivinA is included to monitor the efficiency of DE differentiation; during the mid/hindgut patterning step, a control excluding FGF4 and or WNT3a is used to monitor both CDX2 induction as well as generation of 3-dimensional spheroids.

\section{Applications of the method}

We believe that this culture system will be useful in a wide variety of applications, including functional studies of human intestine development, maturation, and function; studies to understand factors controlling intestinal epithelial proliferation, differentiation and renewal; a platform for high throughput analysis of drug transport and uptake studies; and infectious disease studies. In addition, using patient specific induced pluripotent stem cells (iPSCs) will allow in vitro disease modeling to study the etiology and molecular mechanisms of disease processes. Lastly, we think that this system may be a starting point for tissue replacement therapies either by direct engraftment into diseased bowel or using bioengineering approaches to generate intestinal segments in vitro.

\section{Comparison with other methods}

Several groups have demonstrated that mouse embryonic stem cells and induced pluripotent stem cells have the capability to generate cells that express intestinal markers ${ }^{9-12}$. However, these methods utilize spontaneous differentiation of pluripotent stem cells into embryoid bodies (EB), which contain many cell types derived from all three primary germ layers ${ }^{13}$. More recently, it has been shown that endoderm and intestinal generation can be enhanced in EBs by using a two-step differentiation protocol ${ }^{14}$. 
Our differentiation method represents a significant advance over previous protocols for several reasons; 1) This is the first demonstration of differentiation of human PSCs into intestinal tissue, 2) The protocol directs differentiation of PSCs through stages that mimic embryonic intestinal development and is highly efficient, resulting in organoids containing an epithelium, of which, more than $90 \%$ expresses CDX2 3) Three dimensional growth results in intestinal tissue with both secretory and absorptive function, 4) Organoids contain all of the intestinal epithelial cell types and similar architecture with villus- and crypt-like structures, 5) Organoids contain a stratified mesenchyme and express markers of smooth muscle and intestinal sub-epithelial myofibroblast (ISEMF) cells.

The method described herein does not rely on the inherent ability of ES cells to spontaneously differentiate into all tissue lineages. Furthermore, this is the first protocol to describe a defined set of growth factors that are required at each stage of differentiation to mimic embryonic development in vitro.

Despite offering such great potential, this system is not without its limitations. For example, the intestinal organoids lack several components of the intestine in vivo, such as the enteric nervous system as well as the vasculature, lymphatic and immune systems. Additionally, while all of the major epithelial cell types are generated in proportions similar to those found in vivo, and there is evidence of crypt-like domains housing stem cells, the 3-dimensional architecture is not as regular as is seen in vivo and the villus-like structures are variable from one organoid to the next.

Regardless of these drawbacks we believe that this system has extraordinary experimental utility for understanding and modeling human intestinal development, homeostasis, and disease. Moreover this system provides a viable starting point for future efforts at bioengineering human intestine.

\section{Materials}

\section{Reagents}

\section{Cells}

- H1 or H9 human embryonic stem cells (Wicell International Stem Cell Bank, Wicell Research Institute)

- Induced pluripotent stem cells (Generated by the Pluripotent Stem Cell Facility, Cincinnati Children's Hospital)

\section{Growth media and supplements}

- $\quad$ mTeSR1 media (Stem Cell Technologies, 05850)

- $\quad$ Advanced DMEM:F12 (Invitrogen, 12634-010)

- $\quad$ RPMI Medium 1640 (Invitrogen, 11875)

- $\quad$ hESC qualified Matrigel (BD Biosciences, 354277)

- $\quad$ Matrigel (Intestinal Matrigel)(BD Biosciences, 354234)

- $\quad$ Defined fetal bovine serum (dFBS) (Hyclone, SH30070.02)

- $\quad$ L-glutamine (100×) (Invitrogen, 25030-081)

- Pen/Strep (100×) (Invitrogen, 15140-122)

- $\quad 50 \times$ B27 supplement (Invitrogen, 17504044) 
- Hepes Buffer (Invitrogen, 15630080)

\section{Enzymes and Growth Factors}

- Dispase (Invitrogen, 17105-041)

- Activin A (R\&D Systems, 338-AC)

- $\quad$ FGF4 (R\&D Systems, 235-F4)

- $\quad$ WNT3a (R\&D Systems, 5036-WN)

- $\quad$ Noggin (R\&D Systems, 6057-NG)

- Rspondin1 (R\&D Systems, 4645-RS)

- $\quad$ EGF (R\&D Systems, 236-EG)

CRITICAL: We have not tested growth factors from other vendors.

\section{Immunostaining Reagents}

- Donkey Serum (Jackson ImmunoResearch Laboratories, 017-000-121)

- Donkey anti-goat DyLight 488 (Jackson ImmunoResearch Laboratories, 705-485-147)

- Donkey anti-rabbit Cy3 (Jackson ImmunoResearch Laboratories, 711-165-152

- Donkey anti-mouse DyLight 488 (Jackson ImmunoResearch Laboratories 715-485-150)

- Goat anti-Sox17 (R\&D Systems, AF1924)

- Rabbit anti-FoxA2 (Kind gift from Dr. Jeff Whitsett)

- Mouse anti-Cdx2 (Thermo Scientific/Lab Vision, RM-2116)

- Draq5 (Cell Signaling Technology, 4084S)

- Fluoromount-G fluorescent mounting media (Southern Biotech, 0100-01)

\section{Other reagents and chemicals}

- Sterile PBS (Sigma, P5368)

- $\quad$ Triton-X 100 (Sigma, T8787)

\section{Equipment}

- Pipet-aid AP cordless motorized serological pipettor (Drummond, 4-000-101)

- Pipetman single channel pipettes (Gilson, 2uL: F144801, 20uL: F123615, 200uL: F123601, 1000uL: F123602)

- $\quad 4 f t$ purifier horizontal clean bench (Labconco, 3612504)

- $\quad$ Stereomicroscope (Leica, MDG 33)

- $\quad$ Stereomicroscope (Leica, MZ FLIII)

- Forceps and/or tungsten needle

- Nunclon delta surface 6 well tissue culture dish (Nunc, 140675)

- Nunclon delta surface 24 well tissue culture dish (Nunc, 142475)

Nat Protoc. Author manuscript; available in PMC 2014 January 20. 
- Nunclon delta surface 4 well tissue culture dish (Nunc, 176740)

Critical: It is critical to use Nunclon delta surface tissue culture dishes to plate the spheroids/matrigel in a "bead" that will allow 3-dimensional expansion of the tissue. Use of other tissue culture surfaces will not allow the matrigel "bead" to form and the matrigel will spread into a thin layer across the surface.

- Disposable scalpel, size 15 (Exelint, 29556)

- $5 \mathrm{ml}, 10 \mathrm{ml}$ serological pipettes (Falcon, 5mL: 357543, 10mL: 357551)

- $20 \mathrm{uL}, 200 \mathrm{uL}, 1000 \mathrm{uL}$ sterilized filter pipette tips

- $\quad 50 \mathrm{~mL}$ conical tubes (Falcon - 352070)

- $1.5 \mathrm{~mL}$ microcentrifuge tubes

- Millipore 0.22uM sterilization conical tubes (Millipore - SCGP00525)

- Nunc Thermanox plastic coverslips (Nunc, 174969)

- Cell scraper

\section{Reagent Setup}

\section{Human Pluripotent Stem Cell lines (hESC and iPSC)}

Grow pluripotent stem cells in feeder free conditions on hESC qualified matrigel as previously described ${ }^{15}$ (National Stem Cell Bank Protocols, www.wicell.org). Briefly, hPSCs are cultured on hESC-qualified, matrigel-coated nunclon delta surface plates (6wells) in a 5\% $\mathrm{CO} 2$ incubator at $37^{\circ} \mathrm{C}$. Cells are passaged onto new plates every $4-5$ days using dispase $(1 \mathrm{mg} / \mathrm{mL})$, as described by National Stem Cell Bank Protocols, www.wicell.org.

\section{Dispase}

Resuspend dispase in Advanced DMEM:F12 to a final concentration of 1mg/mL. Filter sterilize using a Millipore filter sterilization tube, make $10 \mathrm{~mL}$ aliquots and store aliquots at $-20^{\circ} \mathrm{C}$ for up to 6 months.

\section{Aliquoting Matrigel for hPSC culture}

Thaw hPSC qualified matrigel on ice, or overnight at $4^{\circ} \mathrm{C}$. Chill sterile microcentrifuge tubes in a microcentrifuge rack at $4^{\circ} \mathrm{C}$ for 1 hour prior to aliquoting matrigel. Aliquot matrigel into cold microcentrifuge tubes and store at $-80^{\circ} \mathrm{C}$ for up to 6 months. CRITICAL: Matrigel will solidify at room temperature $\left(20-25^{\circ} \mathrm{C}\right)$, so it is important to work quickly and keep matrigel cold throughout the aliquoting process.

\section{Matrigel coating plates for hPSC culture}

Coat 6 or 24 well Nunclon delta surface plates with hESC-qualified matrigel as described by manufacturer. Briefly, thaw matrigel on ice (final dilution is determined by the manufacturer and is lot-dependent) and resuspend in cold Advanced DMEM:F12 media. Add enough cold media+matrigel to the tissue culture plate so that the entire surface is covered $(1 \mathrm{~mL} /$ well for a 6 well and $0.5 \mathrm{~mL} /$ well for a 24 well plate). Incubate plates at room temperature for at least 1 hour prior to plating hPSCs, but plates can be stored at $4^{\circ} \mathrm{C}$ for up to one week.

CRITICAL: Matrigel will solidify at room temperature, so it is important to add matrigel to cold media prior to coating plates. 


\section{Matrigel for Intestine culture (Intestinal matrigel)}

Thaw bottle of matrigel on ice or at $4{ }^{\circ} \mathrm{C}$. Once matrigel has thawed, add B27 supplement (final concentration 1×), Rspondin1 (final concentration 500ng/mL), Noggin (final concentration 100ng/mL) and EGF (final concentration $100 \mathrm{ng} / \mathrm{mL}$ ). Mix well by pipetting. Keeping matrigel cold at all times, make $50 \mathrm{uL}$ aliquots into $1.5 \mathrm{~mL}$ microcentrifuge tubes and store at $-80^{\circ} \mathrm{C}$ for up to 6 months. CRITICAL: We have found that adding growth factors to matrigel is not critical, but does increase the efficiency of spheroid outgrowth.

\section{Growth Factor Reconstitution}

Reconstitute growth factors in $1 \times$ PBS to the following final concentrations: Activin A 100ng/uL, EGF 50ng/uL, FGF4 500ng/uL, WNT3a 500ng/uL, Rspondin1 500ng/uL, Noggin 50ng/uL. After reconstitution, growth factors can be stored at $4{ }^{\circ} \mathrm{C}$ for up to one week. For long-term storage (up to 6 months), make aliquots of growth factors and store at $-80^{\circ} \mathrm{C}$. After thawing an aliquot of growth factor from $-80^{\circ} \mathrm{C}$, store at $4^{\circ} \mathrm{C}$ for up to one week. We do not re-freeze growth factors once thawed.

\section{Day 1 Endoderm Differentiation Media}

Combine RPMI 1640, L-glutamine (final concentration $2 \mathrm{mM}$ ), Pen/Strep (final concentration pen $100 \mathrm{Units} / \mathrm{mL}$; strep $100 \mathrm{ug} / \mathrm{mL}$ ), Activin A (final concentration 100ng/ $\mathrm{mL}$ ). Endoderm differentiation media is best if made fresh each day, but can be stored at $4^{\circ} \mathrm{C}$ for 1-3 days. CRITICAL: No serum used in media on day 1.

\section{Day 2 Endoderm Differentiation Media}

Combine RPMI 1640, 0.2\% dFBS (vol/vol) L-glutamine (final concentration $2 \mathrm{mM}$ ), Pen/ Strep (final concentration pen 100 Units/mL; strep $100 \mathrm{ug} / \mathrm{mL}$ ), Activin A (final concentration $100 \mathrm{ng} / \mathrm{mL}$ ). Endoderm differentiation media is best if made fresh each day, but can be stored at $4^{\circ} \mathrm{C}$ for $1-3$ days.

\section{Day 3 Endoderm Differentiation Media}

Combine RPMI 1640, 2\%dFBS (vol/vol) L-glutamine (final concentration $2 \mathrm{mM}$ ), Pen/Strep (final concentration pen 100 Units $/ \mathrm{mL}$; strep $100 \mathrm{ug} / \mathrm{mL}$ ), Activin A (final concentration $100 \mathrm{ng} / \mathrm{mL}$ ). Endoderm differentiation media is best if made fresh each day, but can be stored at $4^{\circ} \mathrm{C}$ for $1-3$ days.

\section{Mid/Hindgut differentiation media}

Combine RPMI 1640, 2\%dFBS (vol/vol) L-glutamine (final concentration $2 \mathrm{mM}$ ), Pen/Strep (final concentration pen $100 \mathrm{Units} / \mathrm{mL}$; strep $100 \mathrm{ug} / \mathrm{mL}$ ), FGF4 (final concentration 500ng/ $\mathrm{mL}$ ) and WNT3A (final concentration 500ng/mL). Make Mid/Hindgut differentiation media fresh on the first day of induction and store at $4{ }^{\circ} \mathrm{C}$ for up to 4 days.

\section{Intestine growth media}

Combine Advanced DMEM:F12, B27 supplement $(1 \times$ final dilution $=2 \mathrm{mLs}$ per $50 \mathrm{~mL}$ media), L-glutamine (final concentration $2 \mathrm{mM}$ ), Pen/Strep (final concentration pen 100 Units $/ \mathrm{mL}$; strep $100 \mathrm{ug} / \mathrm{mL}$ ), HEPES buffer (final concentration $15 \mathrm{mM}$ ), Rspondin1 (final concentration 500ng/mL), Noggin (final concentration 100ng/mL), EGF (final concentration $100 \mathrm{ng} / \mathrm{mL}$ ). Intestine growth media is best if made fresh, but can be stored at $4^{\circ} \mathrm{C}$ for up to one week. 
PBST

PBS $+0.5 \%(\mathrm{v} / \mathrm{v})$ Triton-X 100. PBST can be made ahead of time and stored at room temperature for up to 6 months.

\section{Immunostaining Blocking Buffer}

Add $10 \%$ donkey serum to PBST (for example, 100uL donkey serum to 900uL PBST). Make fresh before each use and keep on ice.

\section{Procedure}

\section{Passaging hPSCs - Timing 2 hours}

1 At least one hour prior to passaging hPSCs, coat a 24 well Nunclon delta surface tissue culture plate with matrigel as described in "Reagent Setup"

2 Start with a 6-well dish of cells that are $~ 85-90 \%$ confluent and are largely differentiation free (Figure 1b). If spontaneous differentiation is visible, this tissue can be manually removed using a tungsten needle or pulled Pasteur pipette. Aspirate mTeSR1 from the 6-well dish of hPSCs.

Critical Step: The starting density of the 6-well dish is critical to obtain optimal results in the following steps.

3 Cover each well with $1 \mathrm{~mL}$ of dispase and place into incubator at $37^{\circ} \mathrm{C}$ until the edges of colonies start to lift.

Critical Step: Dispase treatment must be optimized for each lot as the enzymatic activity varies from lot to lot.

4 Wash wells 3 times with warm Advanced DMEM:F12.

5 After the third wash place $3 \mathrm{~mL}$ of warm mTesR1 into each well and dislodge colonies from the plate using a sterile cell scraper.

$6 \quad$ Withdraw media containing hPSC colonies from dish using a $10 \mathrm{~mL}$ serological pipette. Triturate colonies by forcefully expelling media and hPSCs from the serological pipette back into the tissue culture dish so that the colonies will be broken into smaller pieces. Repeat this 3-5 times until colonies are broken into the desired size.

Critical Step: We have determined that it is optimal to break colonies into pieces that are $1-2 \mathrm{~mm}$ in diameter prior to plating onto 24 -well dishes.

7 Passage titurated colonies from the 6-well dish into a matrigel-coated 24-well dish at a 1:6 ratio. That is, each well from the 6-well dish, containing $3 \mathrm{~mL}$ of media+hPSCs will be plated into six wells of a 24-well dish, with each well receiving $0.5 \mathrm{~mL}$ of media+hPSCs. Optimally, 5,000 triturated hPSC colony pieces are aliquoted per well (Figure 1c-e).

CRITICAL STEP: Optionally, sterile, plastic coverslips can be placed in 24-well dish prior to matrigel coating so cells can be removed for subsequent immunostaining and analysis with confocal microscopy. Using plastic, not glass, coverslips is critical to allow hPSC attachment and growth.

8 Ensure that hPSC colonies are evenly distributed in the wells by gently tapping on the side of the 24-well dish 5-10 times. Confirm even distribution under a stereomicroscope. Place the 24 well dish in the $37^{\circ} \mathrm{C}$ incubator overnight. 


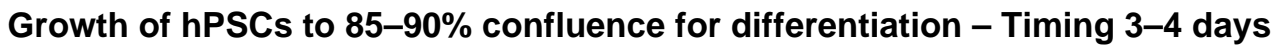

9 The next day, observe the 24-well dish with hPSCs under a stereomicroscope. Do not be alarmed if much of the material passaged the day before appears dead and floating in the media.

10 Aspirate the media and any floating debris from each well with a sterile Pasteur pipette.

11 Add $0.5 \mathrm{~mL}$ of fresh, prewarmed mTeSR1 to each well. Upon further observation, remaining hPSC colonies of a similar size should be uniformly distributed throughout the well (Figure 1c-e). TROUBLESHOOTING

Critical step: Uniform distribution of hPSCs is critical to obtain a $\sim 85-90 \%$ confluent layer of hPSC colonies with minimal spontaneous differentation (Figure 1f-h). Plating cells too densely will lead to premature spontaneous differentiation (Figure 1f, arrows). Similarly, if starting densities are too sparse, adequate confluency may never be reached.

12 Feed 24-well dish with $0.5 \mathrm{~mL} /$ well of fresh mTesR 1 and observe colony density daily. hPSC colonies will reach $\sim 85-90 \%$ confluency for differentiation within 2-4 days.

Critical step: If colony density of the 24-well plate is too dense or too sparse after 2-4 days, discard the plate and start over as differentiation will be inefficient and yield poor results (Figures $1 \mathrm{f}$ and i, Fig. 2). If colonies are too dense at the start of endoderm differentiation, we observe regions that appear undifferentiated at the end of the DE stage (Figure 1i).

\section{Differentiating hPSCs into human definitive endoderm - Timing 3 days}

13 After hPSC colonies have reached $\sim 85-90 \%$ confluence, aspirate mTeSR1 media.

14 To each well, add 0.5mL prewarmed "Day1 Endoderm Differentiation Media" and place the plate back in the incubator.

CRITICAL STEP: After removing mTesR1, it is not necessary to wash cells with prewarmed RPMI 1640 media prior to starting endoderm differentiation.

15 After 24 hours, aspirate "Day1 Endoderm Differentiation Media" and replace with $0.5 \mathrm{~mL} /$ well of prewarmed "Day2 Endoderm Differentiation Media".

CRITICAL STEP: It is normal to observe large amounts of floating cell debris each day during the endoderm differentiation protocol.

16 After 24 hours, aspirate "Day2 Endoderm Differentiation Media" and replace with $0.5 \mathrm{~mL} /$ well of prewarmed "Day3 Endoderm Differentiation Media".

17 After 24 hours, aspirate "Day3 Endoderm Differentiation Media" and wash cells once with "Day3 Endoderm Differentiation Media" without Activin A. Optionally, if hPSCs were plated on plastic coverslips (see Step 7), gently remove plastic coverslip and associated definitive endoderm with forceps to perform immunostaining for FoxA2 and Sox 17 to determine efficiency of DE differentiation as described in Box 1.

CRITICAL STEP: Upon observing cells under a microscope, a flat, confluent sheet of definitive endoderm should be present (Figure 1j). This tissue should contain very little 3-dimensional structure. 
Critical Step: The endoderm should be confluent after differentiation. If cells are too dense $\mathrm{mid} / \mathrm{hindgut}$ spheroids will not form (Figure $2 \mathrm{~g}$ and $\mathrm{j}$ ). If cells are too sparse, mid/hindgut spheroids may form, but will do so with a lower efficiency (Figure 2i and 1) and will not efficiently form intestinal organoids.

TROUBLESHOOTING

\section{Box 1}

Immunostaining to determine efficiency of DE differentiation - Timing 2 days

1 Remove plastic coverslip from 24-well dish containing cells and place in a new 24-well dish.

2 Wash cells once with $0.5 \mathrm{mLs}$ of $1 \times$ PBS.

3 Aspirate PBS and fix cells with $0.5 \mathrm{mLs}$ of $4 \%$ Paraformaldehyde (wt/vol) for 10 minutes at room temperature.

4 Aspirate paraformaldehyde and wash cells 3 times for 5 minutes each wash with $0.5 \mathrm{mLs}$ of $1 \times$ PBS..

$5 \quad$ Aspirate PBS and block cells with $0.2 \mathrm{mLs}$ of "Immunostaining Blocking Buffer" for 30 minutes at room temperature.

6 Aspirate Blocking Buffer.

7 Add primary antibodies to fresh blocking buffer (goat anti-Sox17 1:500, rabbit anti-FoxA2, 1:1000), mix and pipette $0.2 \mathrm{mLs}$ gently over cells.

8 Incubate overnight at $4{ }^{\circ} \mathrm{C}$.

9 The next day, aspirate antibody solution and wash cells 3 times for 5 minutes each wash with $0.5 \mathrm{mLs}$ of $1 \times$ PBS.

10 Aspirate PBS.

11 Add secondary antibodies and Draq5 nuclear stain to fresh blocking buffer (donkey anti-goat DyLight 488 1:500, donkey anti-rabbit Cy3 1:500, Draq5 $1: 1000$ ) mix and pipette $0.2 \mathrm{mLs}$ gently over cells.

12 Incubate at room temperature for 2 hours.

13 Aspirate antibody solution and wash cells 3 times for 5 minutes each wash with $0.5 \mathrm{mLs} 1 \times$ PBS.

14 Remove coverslip with cells and mount on a microscope slide using Fluoromount-G.

15 Visualize cells under a fluorescent microscope. DE will show positive staining for both FoxA2 and Sox17. The percentage of DE present can be quantitated by counting the number of FoxA2/Sox 17 double-positive cells and dividing that number by the total number of Draq5 positive cells (Draq5 is a far-red nuclear stain).

CRITICAL STEP: We routinely observe a differentiation efficiency of 8590\% (SOX17/FOXA2 double positive) $)^{5}$ and we have not determined if spheroids can be generated with a less efficient DE induction. 


\section{Differentiating human DE into human $\mathrm{mid} / \mathrm{hindgut}$ - Timing 3-4 days}

18 Aspirate media from 24-well plate of definitive endoderm and replace with $0.5 \mathrm{~mL} /$ well of prewarmed "Mid/Hindgut differentiation media". Replace with fresh "Mid/Hindgut differentiation media" every 24 hours, for up to 96 hours (4 days).

CRITICAL STEP: Prior to changing the media each day, observe tissue under a stereomicroscope. After approximately 48 hours of exposure to "Mid/Hindgut differentiation media", 3-dimensional structures will start to become visible (Figure $2 \mathrm{~d}-\mathrm{f}$ ). In extremely robust cultures under optimal conditions, it is possible to see free-floating $\mathrm{mid} / \mathrm{hindgut}$ spheroids that have detached from the dish after 48 hours. If free-floating spheroids are present, proceed to Step 19. If no spheroids are present, repeat this Step.

19 Collect spheroids from every well under a stereomicroscope using a 200uL barrier pipette tip and pool approximately 50 spheroids into a $1.5 \mathrm{~mL}$ microcentrifuge tube. Proceed to Step 22 with spheroids.

Critical Step: Pooling approximately 50 spheroids per $1.5 \mathrm{~mL}$ microcentrifuge tube will reduce time spent on subsequent culturing steps.

\section{TROUBLESHOOTING}

20 After collecting spheroids from every well, aspirate remaining media and replace with fresh media as described in Step 18.

21 Immunostaining can be carried out on the epithelial monolayers at the end of 4 days of mid/hindgut induction, following the steps described in Box 1 (use mouse anti-CDX2 (1:500) for Step 7 of Box 1 and donkey anti-mouse DyLight 488 (1:500) for Step 11 of Box 1).

\section{Growing human mid/hindgut spheroids into human intestinal organoids - Timing 1-2 hours to plate $\mathrm{mid} / \mathrm{hindgut}$ spheroids, 14 days to grow tissue}

22 Thaw one aliquot of "Intestinal Matrigel", on ice, per 50 spheroids collected. Allow at least 30 minutes for each aliquot to thaw.

23 After collecting spheroids (Step 19), place microcentrifuge tube upright in a tube rack for 10 minutes. Spheroids should settle to the bottom of the tube by gravity sedimentation.

24 Under a stereomicroscope, observe the spheroids at the bottom of the $1.5 \mathrm{~mL}$ microcentrifuge tube. Using a $200 \mathrm{uL}$ barrier pipette tip, remove up to 50 spheroids from microcentrifuge tube in less than $25 \mathrm{uL}$ total volume.

25 Place spheroids into ice-cold "Intestinal Matrigel" from Step 22 and pipette sample up and down several times to mix. Total volume will be up to $75 \mathrm{uL}$ (50uL Matrigel $+25 \mathrm{uL}$ media+spheroids).

Critical Step: Work quickly to ensure Matrigel does not start to solidify prior to plating.

Critical Step: When pipetting sample up and down to mix, be careful to avoid introducing air bubbles into the matrigel-spheroid suspension.

Critical Step: Use as little media as possible to transfer spheroids into Matrigel. Diluting the Matirgel with too much media will make subsequent steps difficult. 
26 After mixing, remove entire volume using $200 \mathrm{uL}$ barrier pipette tip and slowly pipette into the middle of one well of a 4-well dish. The Matrigel should form a "bead" in the center of the well (Figure 1a, 3a).

Critical Step: After plating Matrigel and spheroids into each well, avoid disturbing the dish for 2-3 minutes to avoid spreading of the Matrigel bead.

Critical Step: Using Nunclon delta surface tissue culture plates is critical for formation of a Matrigel bead. The Matrigel will spread out on dishes from other manufacturers thus limiting 3-dimensional cell expansion.

Critical Step: If air bubbles are present in the matrigel bead, they can be gently removed by aspiration with a sterile $20 \mathrm{uL}$ pipette tip.

CRITICAL Step: A 24-well nunclon delta surface plate can alternatively be used in place of a 4-well plate.

27 After each well of the 4-well dish contains a bead of Matrigel+spheroids, place the dish into the $37^{\circ} \mathrm{C}$ incubator for 10 minutes to allow Matrigel to solidify.

28 Gently pipette $0.5 \mathrm{~mL}$ of "Intestine Growth Media" into each well ensuring that Matrigel/spheroid bead is covered.

29 Replace "Intestine Growth Media" every 4 days, or when the Phenol Red in the media turns yellow.

\section{Splitting human intestinal tissue - Timing 2-3 hours to split tissue, 14 days to grow tissue}

30 After approximately 14 days, reduce the density of tissue in each well by splitting the organoids into fresh Matrigel (Figure 3d,e). To do this, firstly thaw one tube of "Intestinal Matrigel" on ice for every 10-12 organoids that successfully grew during the first 14 days.

\section{TROUBLESHOOTING}

31 Using a sterile scalpel or scissors, trim the tip of a $200 \mathrm{uL}$ pipette to increase the bore size of the pipette such that organoids can pass through the tip without sustaining damage ( $2-3 \mathrm{~mm}$ in diameter).

32 With the cut 200uL pipette tip, dislodge the Matrigel bead containing organoids from each well.

33 Using this tip, vigorously pipette the Matrigel up and down 3-5 times to free organoids from Matrigel.

34 Pool all Matrigel+organoids+media from each well into a sterile Petri dish containing $10 \mathrm{~mL}$ of warm Advanced DMEM:F12. Using a sterile tungsten needle or sterile fine-forceps, dislodge large pieces of matrigel still adherent to the organoids.

35 Under a stereomicroscope, capture up to 6 organoids in the cut 200uL pipette tip and repeat Steps 25-29.

Critical step: Transfer no more than $25 \mathrm{uL}$ total volume (media+organoids) into fresh Matrigel.

36 Culture tissue for 14 days in a tissue culture incubator at $37^{\circ} \mathrm{C}$, replacing "intestine growth media" every 4 days. 


\section{Expanding human intestinal tissue - Timing unlimited}

37 After an additional 14 days in culture (28 total days in culture), thaw one tube of "Intestinal Matrigel" on ice for every 3-4 organoids that successfully grew.

38 Repeat Steps 31-34.

39 Under a stereomicroscope, manually cut organoids into halves using a sterile scalpel. Using a pair of sterile fine-forceps or a tungsten needle to hold organoids in place while cutting will greatly enhance the ease of this step (Figure $3 i$ shows the cutting plane of a 28 day organoid.)

40 Under a stereomicroscope, capture up to 12 organoid halves (6 organoids total) in the cut 200uL pipette tip and repeat Steps 25-29.

41 Cut organoid halves will grow and expand (Figure 3j). Repeat Steps 37-40 every 10-14 days as necessary. We have repeated this expansion for up to 140 days.

\section{Timing}

Splitting hPSCs into 24 well dishes for differentiation. Steps 1-11, 2 hours.

Growth of hPSCs to $85-90 \%$ confluence. Step 12, 4 days.

Differentiating hPSCs into DE. Steps 13-17, 3 days.

Immunostaining. Box 1, 2 days.

Differentiating DE into hindgut. Steps 18-21, 3-4 days.

Growing spheroids into organoids. Steps 22-29, 14 days.

Splitting human intestinal tissue, Steps $30-36,2-3$ hours.

Expanding human intestinal tissue. Steps $37-41$, up to 140 days. The tissue was still viable at 140 days but we have not extended culture past this time point.

\section{Troubleshooting}

Troubleshooting advice is provided in Table 1.

\section{Anticipated Results}

This procedure outlines an extremely efficient method of directed differentiation that generates human intestinal tissue from human pluripotent stem cells in vitro. Each step of the differentiation process yields very specific and robust results. Definitive endoderm differentiation yields a population of cells that is approximately $85-90 \%$ pure by FoxA2+ Sox $17+$ double-positive staining 5 . During the 4-day mid/hindgut induction protocol, morphogenetic tissue movements in the tissue culture dish will give rise to 3-dimensional (3D) structures including epithelial tubes and spheroids. 3D structure is typically first observed at the end of the second day of induction (after 48 hours) and appears mostly as thickenings of the adherent epithelium. Within 2-3 days, spherical structures will begin to delaminate from the adherent epithelium and morphogenesis is complete by the end of 4days at which time spheroids will contain a CDX2+ E-cadherin-positive epithelial layer surrounded by a CDX2+ E-Cadherin-negative mesenchymal layer that is strikingly similar to mouse mid/hindgut development at embryonic day (E) 8.5 (ref. 5). On the third and fourth day of the $\mathrm{mid} / \mathrm{hindgut}$ induction protocol, greater than 20 spheroids are generated per well 
(ranging from 20-42). The robustness of spheroid generation is dependent on the efficiency of endoderm differentiation, and the density/confluency of the endoderm at the end of the 3day endoderm induction step (Figures 1,2).

Using in vitro culture conditions that support the growth and expansion of adult mouse intestinal stem cells ${ }^{6}$, human hindgut spheroids proceed through a series of developmental events that mimics in vivo intestinal development ${ }^{5}$. During the first 14 days in culture, spheroids expand into organoids, giving rise to a pseudostratified epithelium that coexpresses the fetal intestinal transcription factors CDX2, SOX9 and KLF5. Over the next 14 days, the pseudostratified intestinal epithelium transitions into columnar epithelium and gives rise to intestinal tissue that contains an apical-basal orientation including a luminal surface. During this time, villus-like structures emerge into the lumen of the organoids. After approximately one month in culture, human intestinal organoids resemble a fetal mouse intestine, and $\sim 90 \%$ of all epithelial cells express the intestinal marker $\mathrm{CDX}_{2}{ }^{5}$. During prolonged culture ( $>2$ months), villus-like structures emerge and express LGR5 and ASCL2, markers of adult mouse intestinal stem cells ${ }^{6,16}$.

After the first 28 days in culture, organoids can be passaged by manually cutting them in half with a scalpel. After the organoids are cut in half and re-embedded into matrigel, they will continue to grow and tissue will undergo an approximate doubling every 10-14 days. We have successfully passaged organoids for up to 8 passages spanning approximately 140 days, stopping only for an experimental end-point. It is likely that human organoids will continue to grow and expand much longer than this. It is possible that there is a less timeconsuming method by which to split and expand human organoids, however, we have only performed manual passaging to date.

\section{Acknowledgments}

This work was supported by the Juvenile Diabetes Research Foundation JDRF-2-2003-530 (J.M.W.) and NIH, R01GM072915, R01DK080823A1 and S1 (J.M.W.); This work was also supported in part by PHS Grant P30 DK078392 (J.R.S) and K01 DK091415 (J.R.S.). J.C.H. is supported by an Endocrine Fellows Foundation Developmental Research Grant in Diabetes, Obesity and Fat Cell Biology. We also acknowledge core support from the Pluripotent Stem Cell Facility (supported by U54 RR025216).

\section{References}

1. Spence JR, Lauf R, Shroyer NF. Vertebrate intestinal endoderm development. Dev Dyn. 2011; 240:501-520.10.1002/dvdy.22540 [PubMed: 21246663]

2. Wells JM, Melton DA. Early mouse endoderm is patterned by soluble factors from adjacent germ layers. Development. 2000; 127:1563-1572.

3. Dessimoz J, Opoka R, Kordich JJ, Grapin-Botton A, Wells JM. FGF signaling is necessary for establishing gut tube domains along the anterior-posterior axis in vivo. Mech Dev. 2006; 123:4255.10.1016/j.mod.2005.10.001

4. McLin VA, Rankin SA, Zorn AM. Repression of Wnt/beta-catenin signaling in the anterior endoderm is essential for liver and pancreas development. Development. 2007; 134:22072217.10.1242/dev.001230

5. Spence JR, et al. Directed differentiation of human pluripotent stem cells into intestinal tissue in vitro. Nature. 2011; 470:105-109.10.1038/nature09691

6. Sato T, et al. Single Lgr5 stem cells build crypt-villus structures in vitro without a mesenchymal niche. Nature. 2009; 459:262-265.10.1038/nature07935

7. Leibach FH, Ganapathy V. Peptide transporters in the intestine and the kidney. Annu Rev Nutr. 1996; 16:99-119.10.1146/annurev.nu.16.070196.000531

8. Adibi SA. The oligopeptide transporter (Pept-1) in human intestine: biology and function. Gastroenterology. 1997; 113:332-340. 
9. Yamada T, et al. In vitro differentiation of embryonic stem cells into hepatocyte-like cells identified by cellular uptake of indocyanine green. Stem Cells. 2002; 20:146-154.10.1634/stemcells.20-2-146

10. Ishikawa $\mathrm{T}$, et al. Characterization of in vitro gutlike organ formed from mouse embryonic stem cells. Am J Physiol Cell Physiol. 2004; 286:C1344-1352.10.1152/ajpcell.00392.2003 [PubMed: 14960414]

11. Matsuura R, et al. Crucial transcription factors in endoderm and embryonic gut development are expressed in gut-like structures from mouse ES cells. Stem Cells. 2006; 24:624-630.10.1634/ stemcells.2005-0344 [PubMed: 16210401]

12. Konuma N, et al. Mouse embryonic stem cells give rise to gut-like morphogenesis, including intestinal stem cells, in the embryoid body model. Stem Cells Dev. 2009; 18:113-126.10.1089/ scd.2008.0045 [PubMed: 18680392]

13. Axelrod HR. Embryonic stem cell lines derived from blastocysts by a simplified technique. Dev Biol. 1984; 101:225-228. [PubMed: 6692975]

14. Cao L, et al. Intestinal lineage commitment of embryonic stem cells. Differentiation. 2011; 81:110.10.1016/j.diff.2010.09.182 [PubMed: 20934799]

15. Hakala $\mathrm{H}$, et al. Comparison of biomaterials and extracellular matrices as a culture platform for multiple, independently derived human embryonic stem cell lines. Tissue Eng Part A. 2009; 15:1775-1785.10.1089/ten.tea.2008.0316 [PubMed: 19132919]

16. van der Flier LG, Clevers H. Stem cells, self-renewal, and differentiation in the intestinal epithelium. Annu Rev Physiol. 2009; 71:241-260.10.1146/annurev.physiol.010908.163145 [PubMed: 18808327] 


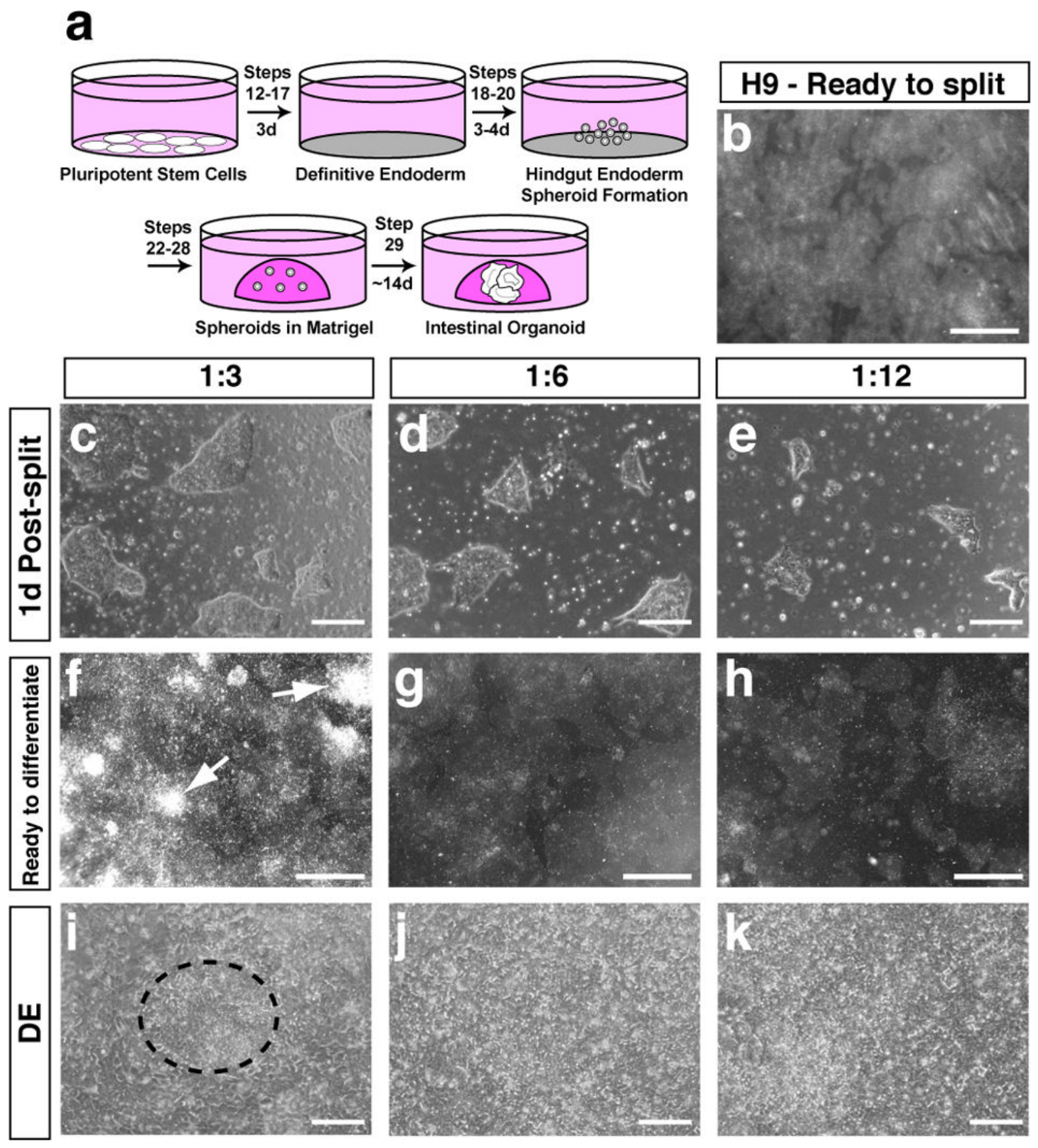

Figure 1.

Passaging hPSCs and differentiation to definitive endoderm. (a.) Schematic representation of the in vitro differentation of intestinal tissue from pluripotent stem cells. (b.) A lowpower image of hPSCs ( $\sim 85-90 \%$ confluent) in a 6-well dish that are ready to passage onto 24-well plates for differentiation. (c-e.) Higher-power images of hPSC colonies one day after plating at different split ratios (wells from 6-well dish: wells in 24-well dish). Cells were plated too dense (1:3) (c), at optimal density (1:6) (d) and too sparse (1:12) (e). (f-h.) Low-power image of hPSCs taken three days after plating. The cells plated at 1:6 dilution (g) are at an optimal density to begin differentiating to definitive endoderm. Note the 
spontaneous differentiation (arrows) in the well plated at 1:3 (f). (i-k.) Monolayers of definitive endoderm at the end of three-day Activin A treatment in different starting densities. Areas that appear to remain undifferentiated (dashed circle) are evident in the cultures that were plated at 1:3 (i). Scale bar in $\mathbf{b}$ and $\mathbf{f}-\mathbf{h}$ corresponds to $2 \mathrm{~mm}$ and in $\mathbf{c}-\mathbf{e}$ and $\mathbf{i}-\mathbf{k}$ to $200 \mu \mathrm{m}$. 

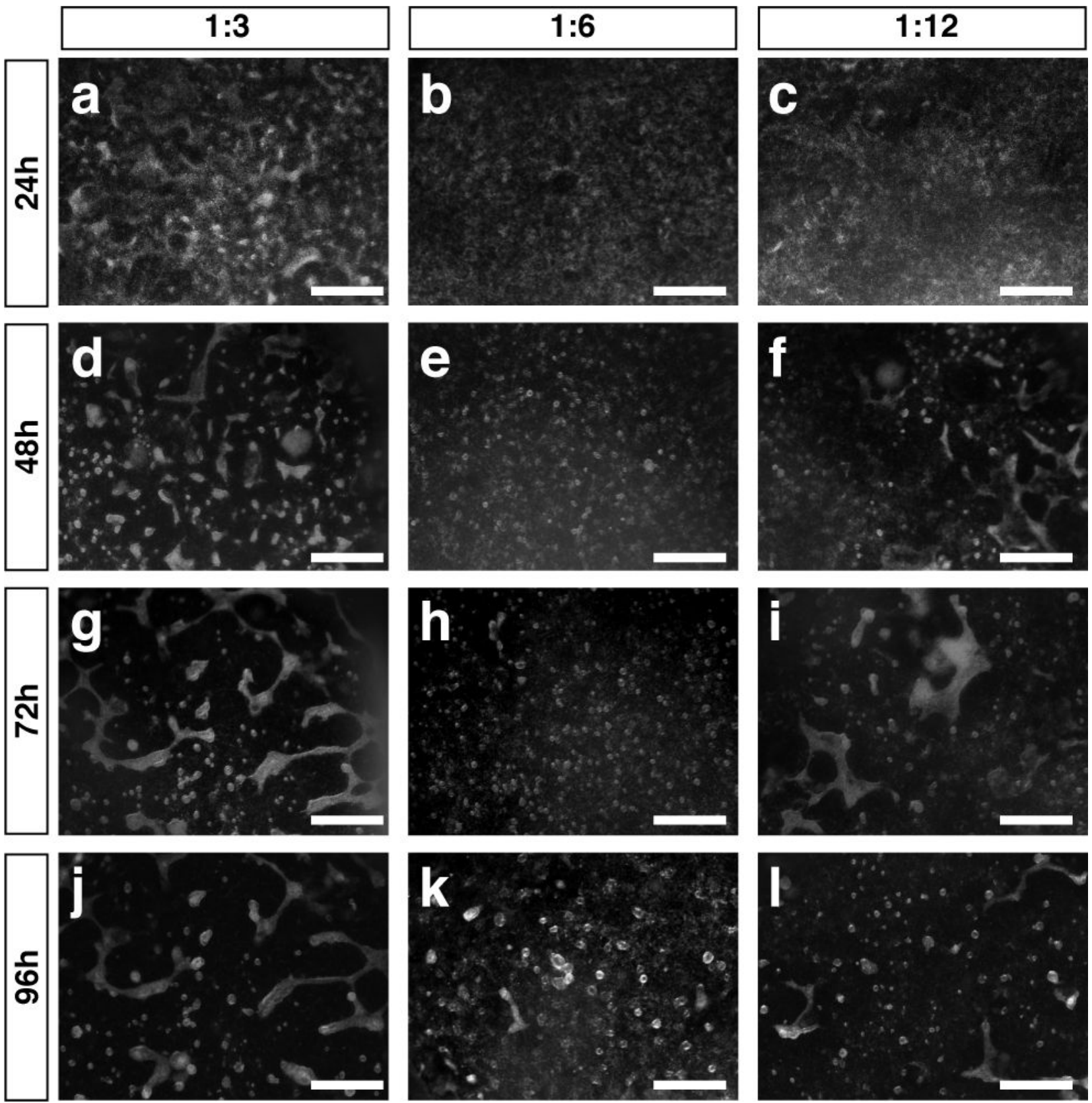

Figure 2.

Spheroid formation from hPSC-derived definitive endoderm. Treatment of endoderm with Wnt3a+FGF4 induces hindgut differentiation and spheroid formation. Low-power images show cultures after $24(\mathbf{a}-\mathbf{c}), 48(\mathbf{d}-\mathbf{f}), 72(\mathbf{g}-\mathbf{i})$ and 96 hours $(\mathbf{j}-\mathbf{l})$ of treatment at different densities, too dense (1:3), optimal density (1:6) and too sparse (1:12). Scale bars correspond to $2 \mathrm{~mm}$. 

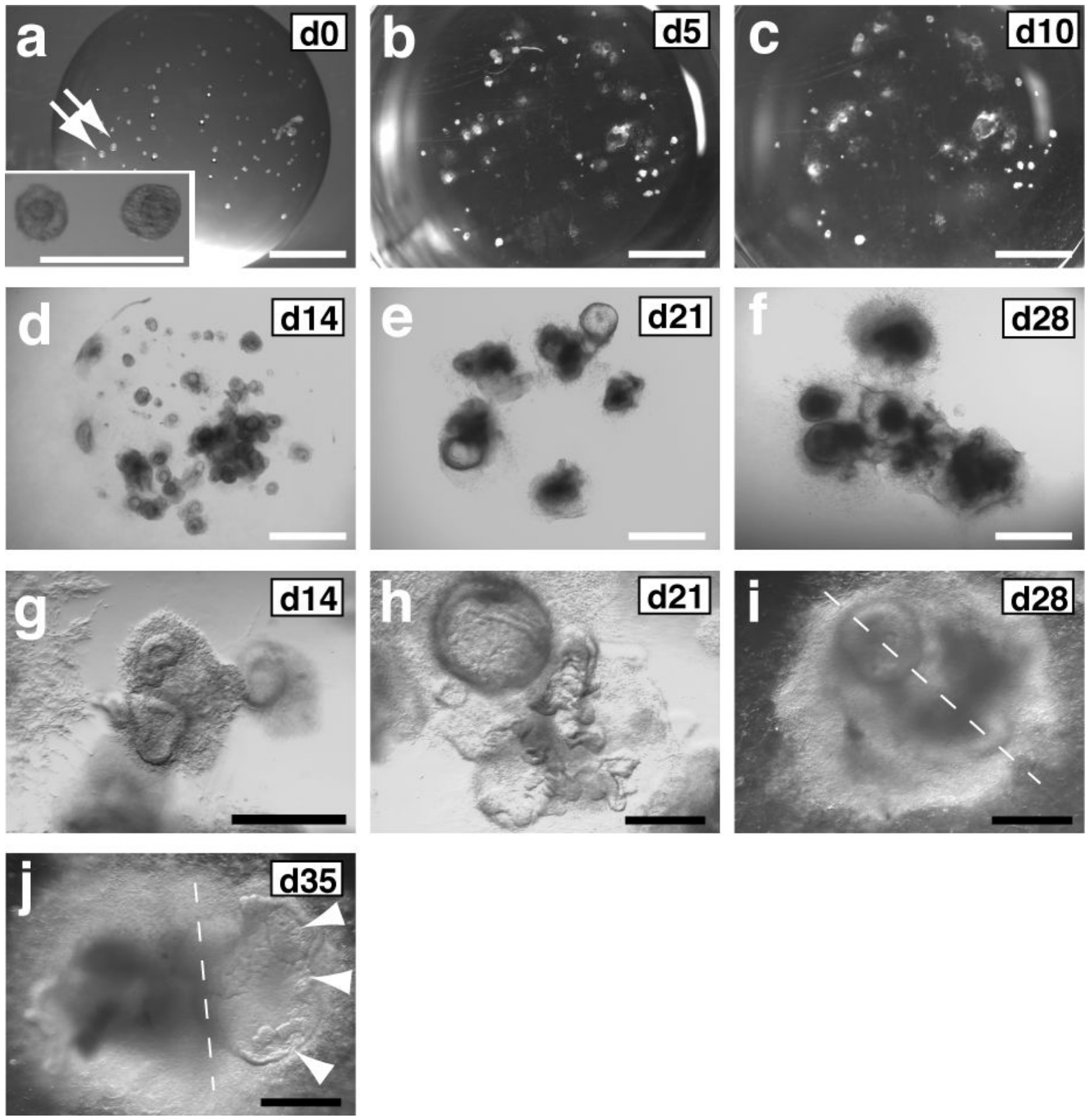

Figure 3.

Three-dimensional growth of intestinal organoids in matrigel. (a-f) Low-power growth series of organoids in matrigel bead. Inset in a shows high-power image of d0 spheroids (arrows). (g-j) High power images of two- to four-week old organoids. (i) A 28 day old organoid that is ready to be split. White dashed line denotes the plane along which the organoid will be cut. (j) A 28 day old organoid that was cut and cultured for an additional 7 days. White dashed line denotes the plane where the organoid was cut, and the arrowheads show new epithelial growth. Scale bar in a-f corresponds to $2 \mathrm{~mm}$ and in $\mathbf{a}$ (inset) and $\mathbf{g}-\mathbf{j}$ to $0.5 \mathrm{~mm}$. 
Table 1

\begin{tabular}{|l|l|l|l|}
\hline STEP & PROBLEM & POSSIBLE REASON(S) & SOLUTION \\
\hline 11 & $\begin{array}{l}\text { Cells not dispersed evenly } \\
\text { throughout dish }\end{array}$ & Not enough agitation to disperse cells & $\begin{array}{l}\text { Tap side of dish several times prior to } \\
\text { placing in incubator (step 8) }\end{array}$ \\
\hline 17 & $\begin{array}{l}\text { Poor DE induction as determined } \\
\text { by Sox17/FoxA2 immunostaining }\end{array}$ & $\begin{array}{l}\text { Cells overgrown prior to DE induction } \\
\text { Bad Activin A }\end{array}$ & $\begin{array}{l}\text { Start induction earlier } \\
\text { Buy new ActivinA or use a fresh aliquot }\end{array}$ \\
\hline 19 & No hindgut spheroids & $\begin{array}{l}\text { Cells too dense } \\
\text { Cells not dense enough } \\
\text { Poor DE induction } \\
\text { Bad growth factors }\end{array}$ & $\begin{array}{l}\text { Determine optimal density prior to starting } \\
\text { differentiation. } \\
\text { Assess DE induction efficiency. } \\
\text { Try new aliquots of growth factors. }\end{array}$ \\
\hline 30 & $\begin{array}{l}\text { Spheroids do not expand into } \\
\text { intestinal organoids }\end{array}$ & $\begin{array}{l}\text { Infrequent media changes } \\
\text { Absence of growth factors/low growth factor } \\
\text { activity }\end{array}$ & $\begin{array}{l}\text { Change media every 3-4 days and ensure } \\
\text { that the growth factors are freshly added. }\end{array}$ \\
\hline
\end{tabular}

